\title{
The development of a conceptual framework to guide sustainable organisational performance
}

\author{
S. Fontannaz and H. Oosthuizen* \\ University of Stellenbosch Business School, University of Stellenbosch, \\ PO Box 610, Bellville 7535, Republic of South Africa
}

Received October 2007

\begin{abstract}
The emergence of the networked economy implies that traditional management approaches no longer suffice in addressing the challenge of complexity. This is compounded by the existence of divergent approaches to determining organisational performance in both management practice and academia, resulting in an execution gap occurring between strategy
\end{abstract} formulation and results.

\begin{abstract}
This article contributes to an understanding of organisational performance by integrating the divergent approaches to determining organisational performance into the Performance 'ESP' framework. This provides a conceptual framework to guide organisational development. The research to validate the framework includes a grounded theory approach, comprising a meta-analytical study of existing research, in-depth qualitative interviews and the pilot testing of the Performance ESP Index, which provides a composite measure of the multi-faceted stakeholder view of organisational performance.
\end{abstract}

The research concludes that organisational performance resides in an organisation's ability to integrate the divergent approaches, to create an execution culture with the necessary dynamic capabilities for sustainable organisational performance in addressing the challenge of complexity. There needs to be diversity in executive abilities at board level to ensure the integration of strategy and people to create the execution culture. Furthermore, leadership should focus on the strategic fusion of strategy and people, whilst management should focus on developing the strategic paradigm throughout the organisation to ensure an execution culture.

The Performance ESP framework provides a diagnostic tool to assess the existence of an execution culture to address the challenge of complexity. The purpose of the assessment tool is to complement the financial metrics of profitability, to ensure a balance between short term profitability and growth for sustainable organisational performance.

Further research is required to confirm the reliability of the Performance ESP index as the initial pilot study, whilst indicative of the potential of the instrument, did not provide conclusive evidence of reliability.

*To whom all correspondence should be addressed.

\section{Introduction}

Organisations can no longer rely on an existing competitive advantage for sustainable performance, as the emergence of the networked economy is resulting in fundamental changes occurring in the business eco-system. Kothuri (2007:1) identifies these changes as an accelerating pace of change, growth in organisational scope and global integration. This has resulted in the networked economy, with the associated growing knowledge-intensity of goods and services and the revolution in information technology.

This perspective is supported by Leibold, Probst, and Gibbert (2002:13), who contend that 'linkages, networks and symbiosis are becoming the order of the day'. The emergence of the networked economy, with the Internet as the most obvious manifestation, has facilitated globalisation, resulting in increased complexity, connectivity and change.
These shifts in the business eco-system are causing hypercompetition across industries, where traditional boundaries are becoming blurred and profitability is being eroded with the deconstruction of traditional value chains (Bresser, Hitt, Nixon \& Henskel, 2000:7). Networking has enabled a degree of bonding throughout the value system, which has fundamentally changed the drivers of profitability.

The implication is that traditional management approaches no longer suffice in addressing the challenge of complexity. This is evidenced by the fundamental shift which has occurred from the industrial age, traditional worldview to the knowledge age, emerging worldview. There is agreement (Wishard, 1995; Dooley, 1997; Dent, 1999) that the traditional worldview no longer applies in the networked economy. Spreitzer and Quinn (2001:Executive summary) confirm this perspective by acknowledging that successful organisations need the knowledge, energy, creativity and ideas of every employee and that the top performing 
organisations achieve results by turning themselves into a company of leaders, where employees at all levels take the initiative and act in the collective interest of the organisation.

This perspective is confirmed by Senge (2006:4) who asserts that 'it is no longer sufficient to have one person learning for the organisation. It's just not possible any longer to figure it out from the top, and have everyone else following the orders of the grand strategist'. According to Senge (2006:4), the organisations that will excel in the future will be the organisations that discover how to 'tap people's commitment and capacity to learn at all levels in an organisation'.

The South African context has the added complexity of balancing conflicting social demands, with the reality of competing in a first world, global arena. The South African economy is underperforming on the projected growth rate of six percent (Business Day, 24/10/2007:2), despite conceptually strong, economic development strategies and an abundance of people. According to Covey (2004:1), there is an execution gap between intended strategy and actual performance, particularly in the public sector.

These fundamental changes in the business eco-system and subsequent challenges to traditional management practice raises questions about the relevance of the existing organisational performance approaches in addressing the challenge of complexity, particularly with regard to the validity and reliability of the established performance management frameworks.

\section{Problem statement}

It appears that organisational development is being undermined by the existence of divergent approaches driving sustainable organisational performance in both management practice and academia. The problem to be addressed in this article focuses on identifying the relevant approach to ensure sustainable organisational performance in the networked economy, which is characterised by the challenges of complexity, connectivity and change.

\section{The research proposition}

The research proposition contends that sustainable organisational performance is dependent on the integration of the divergent approaches to determining organisational performance. Specifically, the necessary prerequisites for organisational performance are the strategic fusion of the strategy and people approaches to ensure a culture of execution. Essentially, execution, strategy and people represent the 'ESP' of organisational performance. The existence of an execution culture is also dependent on individual performance throughout the organisation. The 'ESP' of individual performance includes effectiveness, strength and partnership, which repositions strategy from a process to a paradigm throughout the organisation, guiding individual performance to address the challenge of complexity and change.

\section{The research objectives}

The primary research objective focused on defining the key contributors to organisational performance, with the view to integrating these contributors to provide a systemic view of organisational performance. This research objective was translated into the following secondary objectives to guide the research process:

- Define organisational and individual performance in the networked economy.

- Identify the alternative approaches to determining organisational performance.

- Identify the potential to integrate these divergent organisational performance approaches.

- Develop a conceptual framework for integrating the divergent approaches.

- Validate the conceptual framework.

\section{Research approach and methodology}

The research approach adopted a grounded theory approach, which included a combination of the inductive and deductive, qualitative research approaches. This approach utilised secondary data in the form of a meta-analytical, literature study to identify existing research which had been conducted in the organisational development field. The analysis included the process of conceptualisation, which involved the disaggregation of a mass of data into meaningful and related parts or categories, then rearranging and analysing the data systematically, and rigorously transforming the nature of the data.

This was followed by empirical research, which included indepth interviews and case studies to ground the theory in the reality of management practice and academia. A theoretical, convenience sampling methodology was used, which focused on purposive sampling, to validate specific elements of the theoretical construct. The basis of the sample selection was accessibility and validity, as determined by the preliminary research study. This research focused on a literature search to identify relevant case studies, which were applicable to specific areas of the study. These interviews and case studies included a globally affiliated, supply chain management organisation, a comparative study of people management practice in the financial services industry and the transformation of a public sector organisation. Insights were also gained from leading academics and the author's experience in performance consulting and entrepreneurship.

The case studies focused on specific contexts and it is acknowledged that the findings from these case studies cannot be generalised in a statistical manner and that the interpretation of the data is subject to the author's cognitive context. The detailed results of this empirical research will be discussed in a subsequent article, which is currently being developed for review by the participating organisations, who requested confidentiality for the original 
research. A process of triangulation was employed to validate the theoretical conjecture sourced in the literature review with the empirical research findings. This required constant comparison of the data to develop the conceptual framework. The qualitative research approach was considered relevant in addressing the complex, social framework of this research, where non-linear and mutual causality exists. The research position of participative observer was assumed, based on a social constructionist approach. The unit of analysis for the research included the individual organisation in determining organisational performance and the individual executive in determining individual performance. It is acknowledged that time constraints have impacted on the theoretical saturation of the approach. More details of the research methodology may be obtained from the author.

\section{Research findings}

\section{Defining performance}

In the industrial age, organisational performance was relatively simple to define in terms of the dominant stakeholder, the shareholder. Kotter and Heskett (1992) in Kirby (2005:30 - 39) defined performance in terms of annual growth in net income, average returns on invested capital, and appreciation in the stock price. Zook (2001) in Kirby (2005:30 - 39) focused on a similar combination, including organisations which have grown both revenues and profits and produced shareholder returns in excess of the cost of capital. Collins (2001) relied on cumulative stock returns relative to the general stock market.

Whilst a financial perspective was valid in the industrial economy, where the key driver of organisational performance was the access to financial capital, it no longer suffices in the networked economy. It is now acknowledged that organisational performance is defined by the satisfaction of a range of stakeholder requirements (Kolk, Van der Veen, Pinkse \& Fortanier, 2005). A broader definition of organisational performance is required to represent the multi-stakeholder perspective introduced by Freeman (1984).

This introduces the triple bottom line concept, which is an attempt to address the multi-faceted nature of organisational performance. The contention is that an organisation's performance should be measured across three dimensions: economic, social/ethical and environmental. The premise of the triple bottom line concept is that this responsibility should be measured, calculated, audited and reported, in a manner similar to financial performance (Neely \& Adams, 2007). Kirby (2005:30-39) confirms that the key challenge presented by the multi-stakeholder perspective is in determining a common unit of analysis for measuring stakeholder satisfaction, across a range of dimensions.

The existing research conducted by Kirby (2005:30-39) confirms that, whilst there is no universal agreement in defining organisational performance, there is agreement that sustainable organisational performance is a combination of growth and profitability. For the context of this article, the assumption is made that organisations cannot sustain performance, unless the interests of the stakeholders are satisfied and that the financial measures of profitability, supported with other more intuitive measures provide the best approximation of organisational performance.

Examples of intuitive measures include existing organisational performance frameworks such as Kaplan and Norton's (2001) balanced scorecard framework, the McKinsey 7-S framework (Peters \& Waterman, 1982) and the Burke-Litwin framework developed by French and Bell (1999). The balanced scorecard framework originated as a performance measurement framework, which has now been expanded into a strategy alignment framework to support an execution culture. Whilst this approach provides a valid strategy execution and measurement framework for ensuring stability, the underlying assumptions of rational decision making and determinism present challenges in addressing conditions of hyper-change.

The original integrative 7-S framework, presented by McKinsey (Peters \& Waterman, 1982), has been developed into a more composite, organisational culture diagnostic framework by Nel and others (Abrahams, 2002). French and Bell (1999) attempted to align the divergent organisational performance approaches in the Burke-Litwin framework, which supports the research proposition that fusion is required between strategy and people, to deliver a culture of execution.

These integrative approaches are challenged by D'Aveni and Gunther (1994:236), who contend that environmental turbulence erodes competitive advantage and that these frameworks limit an organisation's ability to deal with change. They propose the New 7-S framework, which comprises a vision for disruption, general capabilities for executing disruption and product/market tactics to deliver disruption.

Organisational performance is the synthesis of individual performance throughout the organisation. This perspective is supported by Spreitzer and Quinn (2001:executive summary), who contend that organisational performance is dependent on individual performance throughout the organisation. In defining individual performance, the metaanalytical research confirmed that individual performance is dependent on effectiveness (Drucker, 1996), strength (Buckingham, 2002) and partnership (Senge, 2006:4). Drucker (1996) confirmed that effectiveness is the specific technology of the knowledge worker in the networked economy. Whilst intelligence, imagination, and knowledge are essential resources, only effectiveness converts them to results. In terms of partnership, Senge (2006:xi) proposes that the fundamental learning units in an organisation are working teams.

\section{Identifying alternative approaches to performance}

Existing research studies (Peters \& Waterman, 1982; Collins, 2001; Collins \& Porras, 2005; Hrebiniak, 2005; Breene \& Nunes, 2007) confirm the existence of divergent approaches to determining organisational performance. These approaches include execution, strategy and people, representing the 'ESP' of organisational performance. The 
execution perspective provides a process based perspective, which focuses on the process of relating as the unit of measurement. This approach contends that it is not possible to act on an entire system, as the system is made up of individuals, who respond in different ways, which implies that change cannot be determined on a rational basis. The central argument of Stacey (2003) is that strategy is the result of the evolving pattern of individual and collective identities, which emerge in the everyday, ordinary interactions which occur between people. In essence, the strategy process is an everyday occurrence, which is impacted on by everyone within the organisation.

This view is supported by Hrebiniak (2005:11), who defines execution as a dynamic and adaptive process, which compensates for unanticipated events. He posits that execution is a key determinant of competitive advantage, as organisations with sound execution have a sustainable competitive advantage which is difficult to imitate.

Bossidy, Charan and Burck (2002:22) support Hrebiniak's view and identify that the heart of execution lies at the intersection of the three core processes: people, strategy and operations. Execution is the missing link or nexus between strategy and people. A leader's job, according to Bossidy et al. (2002), is to integrate strategy, people and execution to ensure organisational performance. The empirical research conducted by the author confirms the necessity for execution, with this theme being repeated in the majority of in-depth interviews conducted with key executives across industry sectors.

The execution approach addresses the reality that the majority of executive decision making occurs in the ad hoc, daily processes that exist in an organisation. These decisions are not limited to a formal, strategy process, following a linear sequence. This approach presumes an empowered workforce, which represents a challenge in the South African context, given the historical circumstances undermining the empowerment of the workforce.

Whilst execution provides a process based approach, the strategy and people approaches provide systemic perspectives of organisational performance. The strategy approach refers to the rational, prescriptive approach to building competitive advantage, which determines organisational performance. This approach focuses on the formal, rational process of formulation, implementation and evaluation and includes the design, planning and positioning schools (Mintzberg, Ahlstrand \& Lampel, 1998). This approach is prescriptive in advocating what must be done by the organisation and is reliant on the underlying assumption of determinism and is found to be effective in conditions of relative stability, as evidenced in the empirical research. However, the empirical research indicated that this approach needs to be supported with a more composite, people approach to address the challenge of change and transformation.

The strategy approach encompasses both the positioning (Porter, 1985) perspective and the resource based (Barney, 1991) perspective in determining competitive advantage and empirical research confirmed that organisations employed a combination of strategic perspectives. According to Harris and Ruefli (2000:587-603), strategy acts as a fulcrum in the deployment of firm resources in the competitive environment, with the aim to generate sustained competitive advantage and this perspective appears to reflect business practice, as evidenced in the in-depth interviews.

The other systemic approach refers to the descriptive, people approach to organisational performance, which includes all elements which influence change within an organisation, particularly leadership, culture and values. The people approach includes the entrepreneurial, cognitive, learning, power, cultural and environmental schools of strategic management (Mintzberg et al., 1998). The focus is on change leadership, which refers to influencing others to enact change that will lead to the achievement of a desired future state (Swart, 2000:220).

The case study of organisational transformation in the public sector highlighted the role that the people approach played in delivering transformation and organisational performance. The empirical research also included a comparative study of organisations in the financial services sector, which confirmed the necessity for a people approach in addressing the challenge of connectivity, which is reflected in the knowledge economy.

\section{Determining the potential to integrate approaches}

The evolutionary frameworks of Melé and Guillén (2006) and Scott (in Peters \& Waterman, 1982) provide insight into the source of the divergence which exists in the organisational performance approaches. These frameworks highlight the gaps which exist between strategy and people, arising from the perception of man as either rational or social actor. Furthermore, the dimension representing an open or closed system confirms the divergence between the externally focused, positioning based approach and the internally focused, resource based approach to strategy development.

A review of these evolutionary frameworks confirms that there is a pendulum effect operating between these divergent approaches. This is resulting in the current shift from an open system, rational approach (positioning based perspective) to a closed system, social approach (resource based perspective), which is undermining organisational performance in the context of market turbulence. Convergence of these divergent approaches appears to be occurring. This is indicative of the integration of the divergent academic fields contributing to organisational development, which is now maturing into a holistic school of thought.

The shift in emphasis from the positioning based approach to the resource based perspective has resulted in the people approach gaining momentum. Further, there is an increased emphasis on corporate responsibility, particularly in the context of the recent corporate scandals, which is resulting in the integration of the multi-stakeholder theory into organisational performance approaches. The empirical research indicates that the repositioning of the people approach as the dominant organisational performance 
approach is undermining the role of strategy in ensuring sustainable organisational performance. This will impact on an organisation's ability to address the challenge of complexity.

It emerges from the research that there are divergent approaches to organisational performance, and the source of this divergence can be traced to the contribution from different academic fields of study to the organisational development field. From the review of the management theory evolution and the findings of the empirical research, it does not appear that the approaches are mutually exclusive and this integration of approaches is supported by others in the academic community (Bridoux, 2004:1). Further, the empirical research highlights the necessity for integrating the approaches to ensure sustainable organisational performance. This confirms the potential for developing a cohesive, integrative framework to guide sustainable organisational performance.

\section{The conceptualisation of the performance ESP framework}

The Performance ESP framework draws on the contention that any effort to understand organisational performance must rest on an underlying theory of the organisation and the associated perspective of strategy. In this regard, it appears that existing research has tended to fragment or dichotomise the important parts of the problem, which is further compounding the problem. This is confirmed by Senge (2006), who asserts that the fragmentation of complex tasks into more manageable fragments results in losing the intrinsic sense of connection. Physicist Bohm (in Senge, 2006) confirms that the task is futile and compares the process to that of reassembling the fragments of a broken mirror to see a true reflection.

The purpose of the Performance ESP framework is to address this fragmented view of organisational performance, by integrating the alternative, process based perspective of execution with the systemic perspectives of strategy and people. The rationale for this methodology is to address the multi-faceted nature of organisational performance. This is achieved by combining the discipline of the rational, prescriptive approach, which focuses on creating stability and organisational alignment, with the more innovative, adaptive process perspective of the descriptive, people approach.

Whilst these approaches assume that the organisation can be enacted upon as a 'whole' system, the process based perspective of execution addresses the fact that the system is comprised of interacting individuals, who respond differently to change initiatives. The challenge of complexity, particularly the assumption of mutual causality, implies that all the variables and interactions between the variables cannot be rigorously drawn. It is acknowledged that this framework provides a constructed reality and the author acknowledges that it is not possible to represent all constructs influencing organisational performance.
The Performance ESP framework has been developed in response to Senge's (2006) contention that the pressure to perform is relentless, whilst the time available for people to think and reflect is becoming scarcer. According to Deming (in Senge, 2006:x), this is exacerbated by the prevailing system of management, which at its core, is dedicated to mediocrity. It forces people to work harder and harder to compensate for failing to tap the spirit and collective intelligence that characterises people working together at their best. The Performance ESP framework and assessment instrument provide time pressured executives with a simple framework to assess organisational and individual performance.

The Performance ESP framework presumes a dynamic environment of punctuated equilibrium, where there are periods of stability interspersed with instability. This phenomenon is occurring in both the external environment and within the organisation. This addresses Breene and Nunes's (2007) contention that context is one of the critical issues in defining organisational performance. Contextual possibilities are described in the framework and inform the choice of organisational approach. Drawing on the work of Stacey (1996:21-48), Figure 1 represents the four dimensions in which organisations operate and the behaviour associated with each dimension.

An explanation of each quadrant, illustrated in Figure 1, is detailed below.

- Traditional: This quadrant represents the context of stability in both the external environment and internal agreement within the organisation, which is represented by the organisational culture.

- Bureaucratic: As the level of change increases in the external environment, there is a tendency for organisations to exhibit strategic inertia, and continue to entrench the level of stability within the dominant culture ('the way things are done').This creates a bureaucratic environment, which undermines organisational performance.

- Innovative: As the pressure for change increases, organisations tend to experience conflict, which leads to a disruption of the status quo. This is a necessary condition, where new ways of doing things are developed, leading to innovation as the organisation adapts to the external environment.

- Political: The nature of organisations and individuals is to converge towards stability. The process is political, as different coalitions promote conflicting positions of stability, until a state of equilibrium is reached, which is represented by the traditional dimension. As complex adaptive organisations, the dynamic nature of the organisation will not be able to sustain the balance, which will generate a renewed spiral of behaviour. 


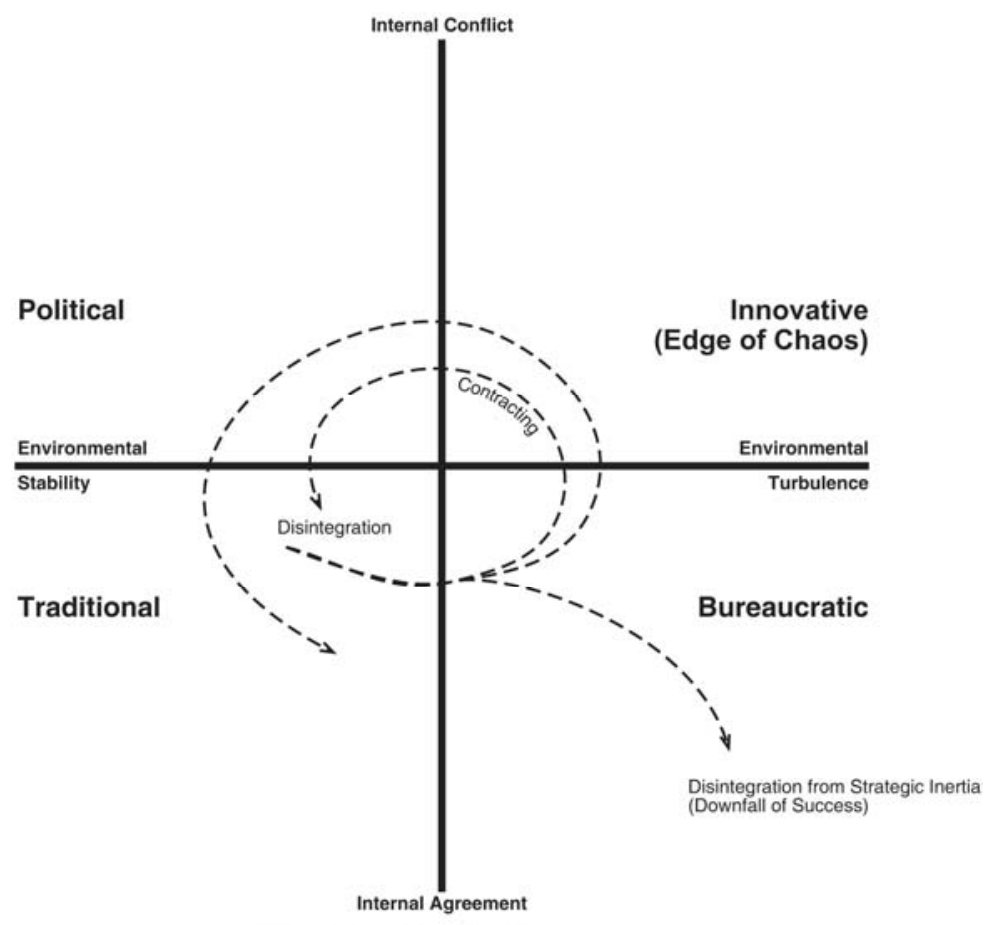

Quadrants of Change

Figure 1: Dimensions of change

Source: Author's conceptual framework, based on the work of Stacey (1996)

These contextual dimensions are relevant, as they determine the dominant organisational approach required by organisations to ensure sustainability. Three points are relevant.

- The context is dynamic, with periods of stability and change in both the external environment and the internal environment, represented by the organisational culture.

- The preferred approach will be determined by the contextual stability or lack thereof.

- Organisational performance requires different organisational approaches at different points in time.

Within the contextual reality of stability and change, the proposed Performance ESP framework consists of four core elements as illustrated in Figure 2.

The constructs of the Performance ESP framework are described in more detail below:

- $\quad$ The circle represents an overview of the organisational performance approaches, which are represented by the emergent, process based approach of execution, and the systemic approaches comprising the prescriptive, strategy and the descriptive, people approach.

- Within the circle are the two triangles, which represent the organisational and individual perspectives of performance. These performance drivers are represented by the Greek symbol of the triangle, which symbolises transformation. The organisational performance triangle, comprises execution, strategy and people (represented by the first letter of each element contributing to the 'ESP'), with strategy and people representing organisational potential and execution representing performance. This supports the research proposition, which contends that organisational performance is dependent on the existence of an execution culture, which requires strategic fusion of the strategy and people approaches.

- The role of leadership focuses on the strategic fusion of strategy and people to ensure sustainable organisational performance.

- The role of management focuses on building an execution culture, which is determined by the existence of a strategic paradigm throughout the organisation.

- $\quad$ The existence of a strategic paradigm is dependent on the level of individual effectiveness and strength, supported by partnership (the 'ESP' of individual performance). Whilst individual strength and partnership represent an individual's leadership potential, it is an individual's level of effectiveness that determines individual performance. The development of a strategic paradigm is particularly relevant in the South African context, where historical factors have inhibited the level of empowerment within organisations. 


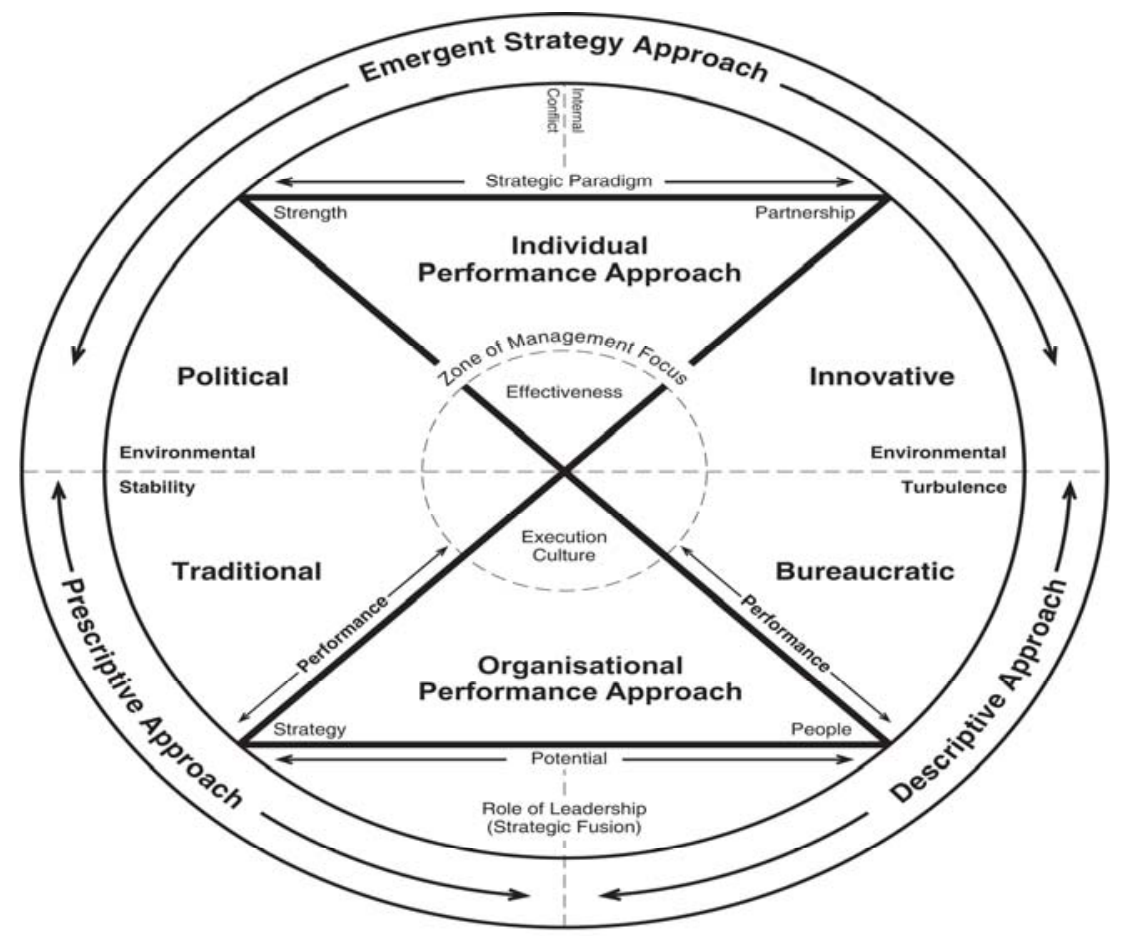

Performance ESP ${ }^{\mathrm{TM}}$ Framework

Figure 2: The performance ESP framework

Source: Author's conceptual framework

The Performance ESP framework addresses the question of which organisational approach is relevant for a particular contextual dimension. In superimposing the triangles (which appear in Figure 2) onto the contextual dimensions of environmental stability/turbulence and internal agreement/conflict (represented in Figure 1), it is possible to identify which organisational approach is relevant for a particular context.

The prescriptive, rational hard 'strategy' approach to organisational performance remains relevant in a traditional environment. In conditions of environmental stability and internal agreement, the prescriptive approach represents the most efficient alternative for achieving organisational performance, provided this approach is customer centric and employs a variety of strategic management analysis tools, to provide a collaborative, systemic view of organisational performance.

The integration of the descriptive, social 'people' approach with the prescriptive, strategy approach is more relevant as the context experiences greater instability, as it is no longer possible to direct organisational behaviour on a rational basis. The role of leadership is particularly relevant in this context, as it is the leader's role to contain anxiety within the group and provide confidence in the new way of acting. Without strong leadership, the organisation will become bureaucratic, which will undermine sustainable performance.

In addressing the challenge of complexity, identified as hyper-change, an emergent strategy approach to organisational performance becomes relevant. This approach does not assume determinism (a fundamentally flawed assumption in conditions of hyper-change). However, this approach can only be implemented in organisations where individuals are empowered and exhibit a strategic mindset to organisational performance.

The Performance ESP framework provides a structured approach to informing organisational development to ensure sustainable performance. Whilst financial measures are indicative of current performance, sustainability requires a more composite measure of organisational performance. The Performance ESP framework balances the conflicting demands of profitability and growth, by providing a counter measure to the established financial metrics. It also provides a framework for conversational processes to develop organisational performance in emergent enquiry.

The purpose of the Performance ESP framework is not to apply/prescribe a solution, but to refocus attention on processes. The underlying assumption of the execution approach, which views organisations as complex adaptive systems, precludes the development of a set of generic prescriptions for organisational development, as every organisation is viewed as unique. Transformation is dependent on the development of new paradigms, which require a different focus of attention to achieve the paradigm shift. 


\section{Validation of the conceptual framework}

The Performance ESP framework has been developed into an assessment instrument, with each divergent approach representing a separate construct, comprising 10 variables. Individual performance is also assessed by measuring effectiveness, strength and partnership, each represented by five variables. The structure of the Performance ESP Index is illustrated in Figure 3, which also highlights the different gaps which arise from the divergent approaches to organisational performance.

\section{Summary}

The emergence of the networked economy and the associated shift in worldviews presents organisations with the challenge of complexity, associated with increasing connectivity and change. Furthermore, financial metrics no longer suffice in determining organisational performance, which has become a paradoxical blend of profitability and growth, to satisfy the diverse range of stakeholders.

The secondary research confirmed the existence of three divergent approaches to this multi-faceted concept of organisational performance. These approaches include the process based approach (execution) and the systemic approaches (the prescriptive, strategy and the descriptive, people approach).

The empirical research confirmed that divergent approaches also exist in management practice and academia and that this divergence creates gaps, which impact on and undermine organisational performance. These gaps include the divergence between the strategy and people approach, the different strategic content approaches, the execution gap between strategy and operations and the management prerogative gap, which relates to strategy remaining an executive function, whilst execution is for staff. It was found that there are no obvious barriers to integrating these approaches and the evolution of management practice indicates that convergence is occurring.

The research indicates that strategy addresses the challenge of complexity, by providing the structure and stability to contain anxiety. To avoid strategic inertia in conditions of accelerating change, this approach needs to be balanced with the people approach to ensure the simultaneous collaboration with the informal, shadow organisation to encourage constructive destruction as a mechanism for driving innovation. The tension between these paradoxes represents the potential for performance, and it is the balancing of these contradictory forces which defines sustainable organisational performance.

The Performance ESP framework provides a conceptual, visual integration of the divergent approaches to organisational performance with the 'ESP' representing the divergent approaches of execution, strategy and people. The framework also provides a perspective of individual performance, which includes effectiveness, strength and partnership as the key elements of the strategic paradigm.

\section{Conclusions}

Organisational performance, in the networked economy, is determined by an organisation's ability to address the central themes of complexity, connectivity and accelerating change. The implication for management is that organisational performance is dependent on the ability to integrate the divergent approaches to organisational performance, to ensure balance between the inherent paradoxes, which govern the emerging worldview.

Whilst the traditional, strategic approach remains relevant and essential in addressing the challenge of complexity, this perspective is insufficient in balancing the challenges of connectivity and change. To ensure organisational performance, the prescriptive, strategy approach needs to be integrated with the more composite, people approach to address the challenges presented by increased connectivity and the repositioning of the knowledge worker as a key driver of organisational performance.

A further implication for management is that the systemic approaches to organisational performance need to be balanced with a process based approach to address the execution gap, which exists between strategy and operations. This conversational process positions strategy as an everyday activity, to address the challenge of accelerating change. By integrating the divergent approaches to organisational performance, an organisation's dynamic capabilities are developed, to ensure that performance can be sustained in different contextual realities.

The integration of these divergent organisational approaches is also required in academia, to ensure that the divergence undermining organisational performance is not perpetuated. The current focus of academia on systemic perspectives of strategy formulation and people management remains relevant, but this approach needs to be balanced with a process based, execution approach focused on the implementation of the systemic perspectives at an operational level.

These conclusions confirm the research proposition, which contends that organisational performance is determined by an execution culture. This execution culture is dependent on the strategic fusion of strategy and people. Furthermore, an execution culture depends on the existence of a strategic paradigm, where strategy becomes a way of thinking throughout the organisation. This perspective repositions strategy from management prerogative to everyone's responsibility. However, this repositioning does not imply that everyone is involved in all elements of strategy. Rather, the implication for management is that every employee needs to understand their contribution to strategy, to ensure their commitment to achieving results. 
Organizational Performance (A1), Environmental Stability (A2), Internal Stability (A3)

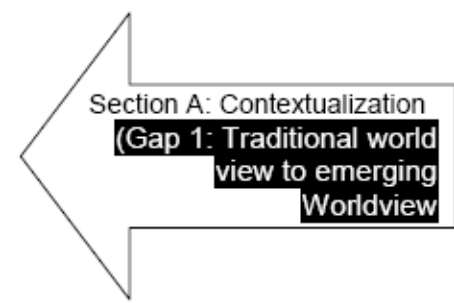

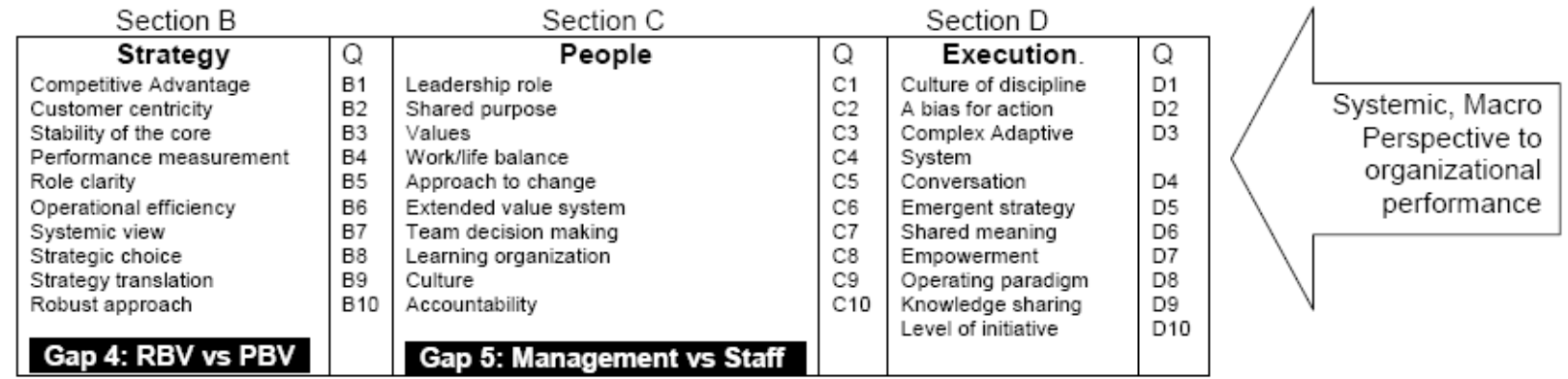

\begin{tabular}{|c|c|c|c|c|c|c|}
\hline Section E & & Section F & & Section G & & \\
\hline $\begin{array}{l}\quad \text { Strength } \\
\text { Core strengths } \\
\text { Prioritization } \\
\text { Dynamic capability } \\
\text { Power influences } \\
\text { Empowerment } \\
\end{array}$ & $\begin{array}{l}\text { Q } \\
\text { E1 } \\
\text { E2 } \\
\text { E3 } \\
\text { E4 } \\
\text { E5 }\end{array}$ & $\begin{array}{l}\quad \text { Partnership } \\
\text { Level of coaching } \\
\text { Team approach to work } \\
\text { Team support } \\
\text { Delegation } \\
\text { Collaboration skills }\end{array}$ & $\begin{array}{l}\text { Q } \\
\text { F1 } \\
\text { F2 } \\
\text { F3 } \\
\text { F4 } \\
\text { F5 }\end{array}$ & $\begin{array}{l}\quad \text { Effectiveness } \\
\text { Personal mastery } \\
\text { Focused results } \\
\text { Level of efficiency } \\
\text { Dominant operating paradigm } \\
\text { Value creation } \\
\end{array}$ & $\begin{array}{l}\mathrm{Q} \\
\mathrm{G} 1 \\
\mathrm{G} 2 \\
\mathrm{G} 3 \\
\mathrm{G} 4 \\
\mathrm{G} 5\end{array}$ & $\begin{array}{r}\text { Process based micro } \\
\text { perspective to } \\
\text { individual } \\
\text { performance. } \\
\text { (Strategy Paradigm) }\end{array}$ \\
\hline
\end{tabular}

Figure 3: Structure of the Performance ESP Index

Source: Author's conceptual framework

The implication for leadership development is that each individual should be encouraged to develop their performance ESP, as represented by the first letters of each of the key elements: effectiveness, strength and partnership. The level of effectiveness refers to an individual's ability to contribute value to the organisation and the other two elements refer to the paradoxical blend of individual strength and collaboration required to deliver results.

\section{Recommendations}

To ensure sustainable organisational performance, it is necessary that the composition of an organisation's board of directors reflect the diversity of skills required to address the challenge of complexity arising from a dynamic environment. The traditional focus on financial skills needs to be tempered with a diverse range of skills, incorporating strategic, people management and entrepreneurial/marketing skills. This will ensure that an organisation can adapt to changing contexts by integrating the skills to ensure strategic fusion of strategy and people and ensuring sufficient innovation to deliver sustainable organisational performance.

The existence of divergent approaches to organisational performance presents time pressured executives with conflicting advice for addressing the challenge of complexity, which undermines the ability to focus on organisational performance. Given the existence of a dominant crises management paradigm, it is proposed that the Performance ESP framework guide organisational development in addressing the challenges of complexity, connectivity and accelerating change. The key role of the leader in the organisation is to ensure strategic fusion of strategy and people, whilst management need to focus on delivering an execution culture.

The development of the Performance ESP index is an attempt to provide time pressured executives with a simple assessment tool to determine the level of integration of these divergent approaches within their organisational context. This tool can also be used as a benchmark to measure the more subjective elements of organisational performance over time. These elements refer to those stakeholder interests, which are not reflected in the financial metrics of the organisation.

This assessment will provide an opportunity to engage in conversational processes, which will refocus attention within the organisation. The underlying premise of the Performance ESP index is that organisational performance requires a paradigm shift, to change behaviour. Without this paradigm shift, behaviour will not change and without a change in behaviour, organisations will not be able to address the challenges of complexity, connectivity and change. 
Whilst the Performance ESP index has been pilot tested as an assessment tool, further research is required to validate the assessment tool. It is proposed that a matched pairs experiment be conducted in different industry sectors to determine the validity and reliability of this measurement instrument. Alternatively, a longitudinal study could be conducted using a sample of organisations, but this study would need to address the problem of constancy of variables and the existence of mutual, non-linear causality, to ensure validity of the results.

\section{References}

Abrahams, M. 2002. 'The beehive of organisational excellence: Entrenching workplace practices that lead to high performance'. Unpublished MBA research report. Bellville: University of Stellenbosch Graduate Business School.

Barney, J. 1991. 'Firm resource and sustained competitive advantage’, Journal of Management, 17:99-120.

Breene, T. \& Nunes, P. 2007. 'Paths to performance: No excuses. Five reasons every company should strive to achieve high performance'. [online] URL: http:// www.accenture.com/outlook. Accessed 12 March 2007.

Bossidy, L., Charan, R. \& Burck, C. 2002. Execution - The discipline of getting things done. London: Random House Business Books.

Bresser, R., Hitt, M., Nixon, R. \& Henskel, D. 2000. Winning strategies in a deconstructing world. New York: John Wiley \& Sons Ltd.

Bridoux, F. 2004. 'A resource based approach to performance and competition: An overview of the connections between resources and competition'. IAG working paper.

Buckingham, M. 2002. Now, develop your strengths. London: Simon \& Schuster UK Ltd.

Business Day, 24 October 2007:2. 'Economy not growing fast enough - Erwin.

Collins, J. 2001. Good to great. London: Random House Business Books.

Collins, J. \& Porras, J. 2005. Built to last. London: Harper Collins.

Covey, S. 2004. 'Business loses momentum at dreaded execution gaps’, Sunday Times Business Times, 11 April:1.

D’Aveni, R. \& Gunther, R. 1994. Hypercompetition. Managing the dynamics of strategic maneuvering. Toronto: Maxwell Macmillan.

Dent, E. 1999. 'Complexity science: A Worldview shift'. A paper submitted to Emergence, George Washington University Administrative Sciences Programme.
Dooley, K. 1997. 'A complex adaptive systems framework of organisational change', Nonlinear Dynamics, Physchology and Life Sciences, 1(1):69-97.

Drucker, P. 1996. The effective executive. Oxford: Elsevier Butterworth-Heineman UK.

Freeman, R. 1984. Strategic management: A stakeholder approach. Marsfield: Pitman.

French, W. \& Bell, C. 1999. Organisation development. Behavioural science interventions for organisation improvement. New Jersey: PrenticeHall.

Harris, I. \& Ruefli, T. 2000. 'The strategy/structure debate: An examination of the performance implications', Journal of Management Studies, 34(4): 587-603.

Hrebiniak, L. 2005. Making strategy work. New Jersey: Pearson Education.

Kaplan, R. \& Norton, D. 2001. The strategy focused organisation. How balanced scorecard companies thrive in the new business environment. Harvard: Harvard Business School Publishing Corporation.

Kirby, J. 2005. 'Toward a theory of high performance', Harvard Business Review, July-August: 30-39.

Kolk, A., Van der Veen, M., Pinkse, J. \& Fortanier, F. 2005. KPMG International Survey of Corporate Responsibility Reporting, June. In the handbook of business ethics and corporate governance. University of Stellenbosch Business School.

Kothuri, S. 2007. 'Knowledge in organisations: Definition, creation and harvesting'. [online] URL: http://www.gse.harvard.edu. Accessed 9 March 2007.

Leibold, M., Probst, G. \& Gibbert, M. 2002. Strategic management in the knowledge economy. New approaches and business applications. Erlangen: Publicis.

Melé, D. \& Guillén,M. 2006. The intellectual evolution of strategic management and its relationship with ethics and social responsibility. Working paper. WP658. IESE Business School. Spain:University of Navarra.

Mintzberg, H., Ahlstrand, B. \& Lampel, J. 1998. Strategy safari: A guided tour through the wilds of strategic management. New York: Free Press.

Neely, A. \& Adams, C. 2007. 'Perspectives on performance: The performance prism'. [online] URL: http://www.som.cranfield.ac.uk/som. Accessed 13 June 2007.

Peters, T. \& Waterman, R. 1982. In search of excellence. 2004 Reprint. London: Profile Books.

Porter, M. 1985. Competitive advantage. New York: Free Press. 
Porter, M. 1991. 'Towards a dynamic theory of strategy', Strategic Management Journal, 12: 95-117.

Senge, P. 2006. The fifth discipline: The art and practice of the learning organisation. London: Random House.

Spreitzer, G. \& Quinn, R. 2001. A company of leaders. San Francisco:Jossey-Bass.

Stacey, R. 1996. Strategic management and organisational dynamics. London: Pitman.

Stacey, R. 2003. Strategic management and organisational dynamics. The challenge of complexity. Harlow: Pearson Education.

Swart, S. 2000. Organisational change. Durban: Butterworths.

Wilshard, W. 1995. 'Business and the new worldview', World Business Academy Perspectives, 9(4).

\section{Personal interviews}

Carreira, P. 2007. Senior manager, process information management process solutions: operations, South African Revenue Service (SARS). Stellenbosch: Telephonic interview, 26 April.

Kirsten, K. 2007. Managing Director, Village of Leaders. Cape Town: Personal interview, 04 May.

Nel, J. 2007. Human Resource Consultant, Old Mutual Business School: Organisational development and change. Cape Town: Personal interview, 17 April.

Van Dyk, L. 2007. Head of the centre of leadership studies, University of Stellenbosch Business School. Bellville: Personal interview, 18 April.

Williams, P. 2007. Managing Director, Safcor Panalpina. Johannesburg: Personal interview, 10 April.

Van Rhyn, L. 2007. Management Consultant, Symphonia Consulting. Bellville: Personal interview, 16 May. 